\title{
NONLINEAR PARABOLIC EQUATIONS AND PROBABILITY
}

\author{
BY D. J. HEBERT, JR.
}

Introduction. The linear parabolic differential equation

$$
\frac{\partial u(t, x)}{\partial t}=\sum_{i j} a_{i j}(t, x) \frac{\partial^{2} u(t, x)}{\partial x_{i} \partial x_{j}}+\sum_{i} b_{i}(t, x) \frac{\partial u(t, x)}{\partial x_{i}}
$$

and its connection with Markov processes with continuous paths, called diffusion processes, has been studied extensively, for example in the books of Doob [3], Itô and McKean [8], Dynkin [2], Mandl [12], Gihman and Skorohod [5], and in the papers of Stroock and Varadhan [15]. The equation (1), called the diffusion equation, is associated with a family $P(t, x)$ of probability measures on the space $C\left([0, \infty), R^{m}\right)$ of continuous $R^{m}$-valued functions on $[0, \infty)$. Each of these measures defines a Markov process $x(s, w)$ with continuous trajectories starting at the point $x$ at time $t$, and the solution $u(t, x)$ of (1) may be represented on an interval $[0, T]$ in terms of its initial value $u(0, x)$ by the formula

$$
u(t, x)=\int u(0, x(T, w)) P(T-t, x)(d w) .
$$

Note that the process $x(s, w)$ is scaled in reverse, i.e. $s=T-t$ for $t \leqq T$.

A common example of a diffusion equation is the Fokker-Planck equation of statistical mechanics for which the solution $u(t, x)$ represents the density in phase space at time $t$ for a fluid particle. This equation is usually derived as a nonlinear equation with coefficients $a_{i j}$ and $b_{i}$ dependent in some way upon the solution $u$ (see [16] for several such derivations). In textbooks, the dependence upon $u$ is neglected to simplify the theory. One approach to the study of the nonlinear equation was introduced by McKean [13], [14] based upon a derivation of Kac [9] which gives coefficients as a function of the value of $u$. Under certain smoothness conditions for the coefficients, McKean showed the existence and uniqueness of a

AMS (MOS) subject classifications (1970). Primary 28A40, 35K55, 47H05, 46G05, 60J60; Secondary 28A30, 60H05, 47H15, 47D05.

Key words and phrases. Nonlinear, parabolic, stochastic process, Markov process, accretive, derivative. 
Markov process $x(t, w)$ which solved an associated stochastic differential equation. The trajectories represented paths of a particle in a "bath" of like particles.

In the present paper we consider a more general nonlinear diffusion equation of the form

$$
\partial u(t, x) / \partial t=A u(t, x)
$$

where

$$
A u=\sum_{i j} a_{i j}\left(x, u, \frac{\partial u}{\partial x}\right) \frac{\partial^{2} u}{\partial x_{i} \partial x_{j}}+\sum_{i} b_{i}\left(x, u, \frac{\partial u}{\partial x}\right) \frac{\partial u}{\partial x_{i}} .
$$

For a given initial density $u(0, x)$, approximations $u_{n}(t, x)$ are formulated in terms of probability measures associated with the derivative of the operator $A$ in the Banach space $C_{0}\left(R^{m}\right)$. The result is that with mild conditions upon the coefficients, the approximations converge to a solution $u(t, x)$ to (3) which has representations in terms of probability measures. This method of proving existence and uniqueness of solutions of the parabolic equation (3) is quite different from the standard methods such as the Galerkin method given in [11] and the method of Sobolevski given in [4]. A new condition for global existence appears naturally in this new approach. The connection with probability theory obtained here shows that most of the linear theory may be applied to the study of nonlinear diffusion equations.

The proofs of the theorems will appear in [6] and [7]. The main existence theorem is a consequence of a general theorem given in [7], whose proof depends upon the concept of "accretive" operator in the sense of Kato [10] and Crandall and Liggett [1]. (For references see these papers.)

Statement of the results. Let $C_{0}\left(R^{m}\right)$ denote the Banach space of continuous real valued functions on $R^{m}$ which vanish at infinity, and let $D$ denote the dense linear subspace consisting of functions whose second derivatives are uniformly Hölder continuous. We seek a solution $u(t, x)$ to the equation (3) when the initial condition $u_{0}=u(0, \cdot)$ is given. The following assumptions are made:

1. The functions $u_{0}$ and $A u_{0}$ are in $D$.

2. The operator $A$ is uniformly elliptic, i.e., there are constants $\lambda$ and $\mu$ such that for each $x, y$, and $p$ in $R^{m}$ and $u$ in $R$,

$$
\lambda|y|^{2} \leqq \sum a_{i j}(x, u, p) y_{i} y_{j} \leqq \mu|y|^{2} .
$$

3. The coefficients $a_{i j}(x, u, p)$ and $b_{i}(x, u, p)$ and their derivatives with respect to $u$ and $p$ are uniformly bounded and uniformly Hölder continuous in all the variables. 
We now define some linear diffusion operators for each $u$ and $w$ in $D$.

$$
\begin{aligned}
L(u) w & =\sum a_{i j}\left(x, u, \frac{\partial u}{\partial x}\right) \frac{\partial^{2} w}{\partial x_{i} \partial x_{j}}+\sum b_{i}\left(x, u, \frac{\partial u}{\partial x}\right) \frac{\partial w}{\partial x_{i}}, \\
L_{1}(u) w & =L(u)(w)+\sum_{k} c_{k}(u) \frac{\partial w}{\partial x_{k}},
\end{aligned}
$$

where

and finally

$$
c_{k}(u, x)=\sum \frac{\partial a_{i j}}{\partial p_{k}} \cdot \frac{\partial^{2} u}{\partial x_{i} \partial x_{j}}+\sum \frac{\partial b_{i}}{\partial p_{k}} \frac{\partial u}{\partial x_{j}}
$$

$$
d A(u) w=L_{1}(u) w+d(u) \cdot w
$$

where

$$
d(u, x)=\sum \frac{\partial a_{i j}}{\partial u} \frac{\partial^{2} u}{\partial x_{i} \partial x_{j}}+\sum \frac{\partial b_{i}}{\partial u} \cdot \frac{\partial u}{\partial x_{i}} .
$$

The operator $d A(u)$ is the derivative of $A$ at $u$. If $v$ maps $[0, T]$ into $D$, let $P(v, t, x)$ and $Q(v, t, x)$ be the probability measures associated with the linear operators $L(v(s))$ and $L_{1}(v(s))$, respectively. Finally, let

$$
R(v, t, x)(d w)=Q(v, t, x)(d w) \exp \int_{T-t}^{T} d(v(s), x(s, w)) d s .
$$

For a given positive number $T$ and a positive integer $n$, we subdivide the interval $[0, T]$ into subintervals of length $r=T / 2^{n}$. The approximations $u_{n}(t, x)$ are defined as follows

for $0 \leqq t \leqq r$ and

$$
u_{n}^{0}(t, x)=u(0, x)+t \int R(u(0, \cdot), t, x)(d w) A u(0, x(T, w))
$$

$$
\begin{gathered}
u_{n}^{1}(t, x)=u_{n}^{1}(r, x) \quad \text { for } t \geqq r ; \\
u_{n}^{k}(t, x)=u_{n}^{k-1}(t, x) \quad \text { for } t \leqq k r ; \\
u_{n}^{k}(k r+t, x)=u_{n}^{k-1}(k r, x)+t \int R\left(u^{k-1}, k r+t, x\right)(d w) A u(0, x(T, w))
\end{gathered}
$$

for $0 \leqq t \leqq r$ and

$$
u_{n}^{k}(k r+t)=u_{n}^{k}(k r+r) \quad \text { for } t \geqq r .
$$

Define $u_{n}(t, x)=u_{n}^{p}(t, x)$ where $p=2^{n}$, so that $u_{n}(t, x)=u_{n}^{k}(t, x)$ for each $k$ when $t \leqq k r$.

The first existence theorem is as follows:

THEOREM 1. If the assumptions 1, 2, 3 hold, then there is an interval $[0, T]$ and a unique mapping $u$ of $[0, T]$ into $D$ such that the functions $u_{n}(t, \cdot)$ converge in $C_{0}\left(R^{m}\right)$ to $u(t)$, uniformly in $t$ and $\partial u(t) / \partial t=A u(t)$. 
An additional hypothesis gives a global existence theorem:

THEOREM 2. If the assumptions $1,2,3$ hold and if the functions $c_{k}(v, x)$ and $d(v, x)$ have a bound independent of $v$, then for each interval $[0, T]$, there is a unique mapping $u$ of $[0, T]$ into $D$ such that the functions $u_{n}(t, \cdot)$ converge in $C_{0}\left(R^{m}\right)$ to $u(t)$ uniformly in $t$ and $\partial u(t) / \partial t=A u(t)$.

Next we see how the solutions $u(t)$ are represented and approximated by probability measures:

THEOREM 3. If the hypotheses of Theorem 1 or Theorem 2 are satisfied so that the approximations $u_{n}(t, \cdot)=u_{n}(t)$ converge to a solution $u(t, \cdot)=$ $u(t)$ of (3), then there is a subsequence $\left(u_{n_{k}}\right)$ such that $Q\left(u_{n_{k}}, t, x\right)$ converges to $Q(u, t, x)$ and $P\left(u_{n_{k}}, t, x\right)$ converges to $P(u, t, x)$ uniformly on compact subsets of $[0, T] \times R^{m}$. Also

and

$$
u(t, x)=u_{0}(t)(x)+\int_{0}^{t} d s \int R(u, T-s, x)(d w) A u_{0}(x(T, w))
$$

$$
u(t, x)=\int P(u, T-t, x)(d w) u_{0}(x(T, w)) .
$$

Thus the solution $u(t, x)$ to the nonlinear equation (3) has a representation (10) which looks like the representation (2) for the solution to the linear equation, and the integrals

$$
\int P\left(u_{n}, T-t, x\right)(d w) u_{0}(x(T, w))
$$

are approximations to the solution $u(t, x)$ of (3). A corollary to the proof of Theorem 3 is that $\int R\left(u_{n_{k}}, T-t, x\right)(d w) A u_{0}(x(T, x))$ converges to

This implies that

$$
A u(t, x)=\int R(u, T-t, x)(d w) A u_{0}(x(T, w)) .
$$

$$
\frac{d}{d t} A u(t, x)=d A u(t) A u(t, x) .
$$

A case of some interest is the one where the coefficients of $A$ are dependent upon $\partial u / \partial x$ but not upon the value of $u$ itself, for example when the diffusion and drift coefficients are dependent on the gradient of the density. In this case, $d A u(t)$ is itself a diffusion operator and $R(u, t, x)=P(u, t, x)$ is a probability measure. The conditions of Theorem 2 for global existence is also simplified since $d(u)=0$. Finally we note that once the solution $u(t, x)$ is known, the coefficients of $A$ are determined along with the measure $P(u, t, x)$, hence all the results of linear diffusion theory can be applied by considering the linear operator $L(u(t))$. 


\section{REFERENCES}

1. M. G. Crandall and T. M. Liggett, Generation of semi-groups of nonlinear transformations on general Banach spaces, Amer. J. Math. 93 (1971), 265-298. MR 44 \#4563.

2. E. B. Dynkin, Markov processes, Fizmatgiz, Moscow, 1963; English transl., Vol. I, Die Grundlehren der math. Wissenschaften, Band 121, Academic Press, New York; Springer-Verlag, Berlin, 1965. MR 33 \#1886.

3. J. L. Doob, Stochastic processes, Wiley, New York; Chapman and Hall, London, 1953. MR 15, 445.

4. A. Friedman, Partial differential equations, Holt, Rinehart, Winston, New York, 1969.

5. I. I. Gihman and A. V. Skorohod, Stochastic differential equations, "Naukova Dumka", Kiev, 1968; English transl., Ergebnisse der Mathematik und ihrer Grenzgebiete, Band 72, Springer-Verlag, New York, 1972. MR 41 \#7777.

6. D. J. Hebert, Nonlinear diffusion (to appear).

7. - - Probability methods and nonlinear analysis, J. Functional Analysis (to appear).

8. K. Itô and H. P. McKean, Jr., Diffusion processes and their sample paths, Die Grundlehren der math. Wissenschaften, Band 125, Academic Press, New York; Springer-Verlag, Berlin, 1965. MR 33 \#8031.

9. M. Kac, Probability and related topics in the physical sciences, Lectures in Appl. Math., vol. 1, Proc. Summer Seminar (Boulder, Colo., 1957), Interscience, New York, 1959. MR 21 \#1635.

10. T. Kato, Accretive operators and nonlinear evolution equations in Banach spaces, Proc. Sympos. Pure Math., vol. 18, part I, Amer. Math. Soc., Providence, R.I., 1970, pp. 138-161. MR 42 \#6663.

11. O. A. Ladyženskaja, V. A. Solonnikov and N. N. Ural'ceva, Linear and quasilinear equations of parabolic type, "Nauka", Moscow, 1967; English transl., Transl. Math. Monographs, vol. 23, Amer. Math. Soc., Providence, R.I., 1968. MR 39 \#3159a, b.

12. P. Mandl, Analytic treatment of one-dimensional Markov processes, Die Grundlehren der math. Wissenschaften, Band 151, Academia, Prague; Springer-Verlag, New York, 1968. MR 40 \#930.

13. H. P. McKean, Jr., A class of Markov processes associated with nonlinear parabolic equations, Proc. Nat. Acad. Sci. U.S.A. 56 (1966), 1907-1911. MR 36 \#4647.

14. - - Propagation of chaos for a class of nonlinear parabolic equations, Stochastic Differential Equations (Lecture Series in Differential Equations, Session 7, Catholic Univ., 1967), Air Force Office Sci. Res., Arlington, Va., 1967, pp. 41-57. MR 38 \#1759.

15. D. W. Stroock and S. R. S. Varadhan, Diffusion processes with continuous coefficients. I, II, Comm. Pure Appl. Math. 22 (1969), 345-400, 479-530. MR 40 \#6641; \#8130.

16. T. Wu, Kinetic equations of gases and plasmas, Addison-Wesley, Reading, Mass., 1966.

Department of Mathematics, University of Pittsburgh, Pittsburgh, PennsylVANIA 15260 